\title{
Characterization of the Golgi scaffold protein PAQR3, and its role in tumor suppression and metabolic pathway compartmentalization
}

This article was published in the following Dove Press journal: Cancer Management and Research

\author{
Lan Lei ${ }^{1,2}$ \\ Zhe-Nan Ling ${ }^{3}$ \\ Xiang-Liu Chen' \\ Lian-Lian Hong' \\ Zhi-Qiang Ling'
}

'Department of Molecular Oncology, Institute of Cancer and Basic Medicine (ICBM), Chinese Academy of Sciences, Cancer Hospital of the University of Chinese Academy of Sciences, Gongshu District, Hangzhou, 310022, People's Republic of China; ${ }^{2}$ The Second Clinical Medical College of Zhejiang Chinese Medicine University, Hangzhou 310053. People's Republic of China; ${ }^{3}$ Department of Clinical Medicine, Medical College, Zhejiang University City College, Hangzhou 310015, People's Republic of China
Correspondence: Zhi-Qiang Ling Department of Molecular Oncology, Zhejiang Cancer Institute, Institute of Cancer and Basic Medicine (ICBM), Chinese Academy of Sciences, Cancer Hospital of the University of Chinese Academy of Sciences, No.I Banshan East Road, Gongshu District, Hangzhou 310022, People's Republic of

China

Email lingzq@zjcc.org.cn

\begin{abstract}
The Golgi apparatus is critical in the compartmentalization of signaling cascades originating from the cytoplasmic membrane and various organelles. Scaffold proteins, such as progestin and adipoQ receptor $(\mathrm{PAQR}) 3$, specifically regulate this process, and have recently been identified in the Golgi apparatus. PAQR3 belongs to the PAQR family, and was recently described as a tumor suppressor. Accumulating evidence demonstrates PAQR3 is downregulated in different cancers to suppress its inhibitory effects on malignant potential. PAQR3 functions biologically through the pathological regulation of altered signaling pathways. Significant cell proliferation networks, including Ras proto-oncogene (Ras)/mitogenactivated protein kinase (MAPK), phosphatidylinositol 3-kinase (PI3K)/protein kinase B (Akt), insulin, and vascular endothelial growth factor, are closely controlled by PAQR3 for physiologically relevant effects. Meanwhile, genetic/epigenetic susceptibility and environmental factors, may have functions in the downregulation of PAQR3 in human cancers. This study aimed to assess the subcellular localization of PAQR3 and determine its topological features and functional domains, summarizing its effects on cell signaling compartmentalization. The pathophysiological functions of PAQR3 in cancer pathogenesis, metabolic diseases, and developmental ailments were also highlighted.
\end{abstract}

Keywords: cell signaling, Golgi apparatus, metabolic regulation, metastasis, PAQR3, tumor suppressor

\section{Introduction}

Cancer represents the number one killer in developed countries and the second deadliest disease in the underdeveloped world, thereby constituting one of the most important health problems worldwide. ${ }^{1}$ The mechanistic targets of signaling pathways play critical roles in cell growth control, with a large number of multiprotein complexes relaying nutrients, energy, and growth factors through metabolic signaling. ${ }^{2}$ The characteristic features of malignancy, such as rapid growth and proliferation, are enhanced by cell metabolic reprogramming in cancer cells, generating additional energy and providing more anabolic molecules for biosynthesis, cell oxidation, and redox state rebalance in cancer cells. ${ }^{3}$ Cancer metabolism represents an important characteristic of tumors, and is significantly altered at every stage of tumorigenesis.

Multiple signaling networks regulate cell fate mechanisms. Cell signaling conveys extracellular signals into the cell, especially the nuclear compartment, causing cell growth, differentiation, and apoptosis. ${ }^{4,5}$ Increasing evidence suggests the 
Golgi apparatus constitutes a critical signaling organelle for multiple networks originating from the cytoplasmic membrane. Hence, subcellular compartmentalization is considered a significant premise in cell signaling. ${ }^{6,7}$

Progesterone and adipoQ receptor (PAQR3), a Golgi organelle membrane protein, is negatively regulated in numerous human cancers and highly involved in malignant potential via various mechanisms. ${ }^{89}$ It was also demonstrated that PAQR3 modulates metabolic homeostasis and participates in the compartmentalization of cell signaling. ${ }^{6}$ PAQR3 may be regulated by environmental factors and DNA methylation through genetic and epigenetic mechanisms. ${ }^{10,11}$ Understanding the pathways that regulate PAQR3 may provide new therapeutic targets for cancers and metabolic diseases.

\section{The PAQR family and PAQR3 gene}

The PAQR protein family comprises transmembrane proteins widely found in multiple species, eg, archaebacteria, Eubacteria, Caenorhabditis elegans, and mammals. ${ }^{12-14}$ The PAQR family is highly conserved, indicating that it is evolutionary critical. ${ }^{12}$ In mammalians, there are 11 PAQR family members (PAQR1-PAQR11). ${ }^{15}$ PAQR proteins comprise seven transmembrane domains with the $\mathrm{N}$ - and $\mathrm{C}$ - termini found intracellularly and extracellularly, respectively, which is topologically different from typical $\mathrm{G}$ protein-coupled receptors (GPCRs) ${ }^{14,16} \mathrm{~A}$ few PAQR proteins have been previously assessed for their roles. Previous studies have shown that PAQR1 and PAQR2 (or AdipoR1 and AdipoR2, respectively) are receptors for adiponectin, which regulates glucose metabolism. ${ }^{17,18}$ PAQR5, PAQR7, and PAQR8 are receptors for progestin, ${ }^{19-23}$ while PAQR10 acts as a steroid receptor. ${ }^{24}$

The Ras/Raf proto-oncogene (Raf)/MAPK/Erk kinase (MEK)/extracellular-signal-regulated kinase (ERK) pathway modulates several important cell functions, such as growth, apoptosis, differentiation, migration, and metabolism. ${ }^{25-27}$ Ras/Raf/MEK/ERK signaling dysregulation is associated with multiple diseases in humans, specifically playing a crucial oncogenic role in cancers. ${ }^{28-31}$ PAQR3, or RKTG (Raf kinase trapping to Golgi), ${ }^{32}$ represents a type III membrane protein whose $\mathrm{N}$-terminal end faces the cytoplasm (Figure 1A). Many PAQR3 portions target the protein to the Golgi apparatus and promote its binding to Raf- 1 in mammalian cells. ${ }^{33}$ The encoded protein belongs to the PAQR family. ${ }^{14,16}$ PAQR3, the firstly reported Golgi protein containing seven transmembrane domains, has demonstrated tumor suppressor effects via inhibition of Raf/MEK/ERK signaling, thereby blunting Raf-1 signaling to downstream effectors. ${ }^{32-35}$ PAQR3 negatively regulates malignant cell growth and migration, and inhibits angiogenesis in endothelial cells via modulation of the autocrine function of vascular endothelial growth factor. ${ }^{34-37}$ PAQR3 also plays a vital role in carcinogenesis, tumor migration, and epithelial-mesenchymal transition (EMT) via functional interaction with $\mathrm{p} 53 .{ }^{36}$

\section{Topological structure and subcellular localization of PAQR3}

The PAQR3 gene is distributed in the whole eukaryotes, and it has an ancient evolutionary history. From the evolutionary tree, it differentiated from the PAQR gene family earlier and has a relatively independent differentiation direction, which is different from the PAQR10 and PAQR11 belonging to the differentiation branches of hemolysin class 3 proteins (hemolysin III type protein,

A

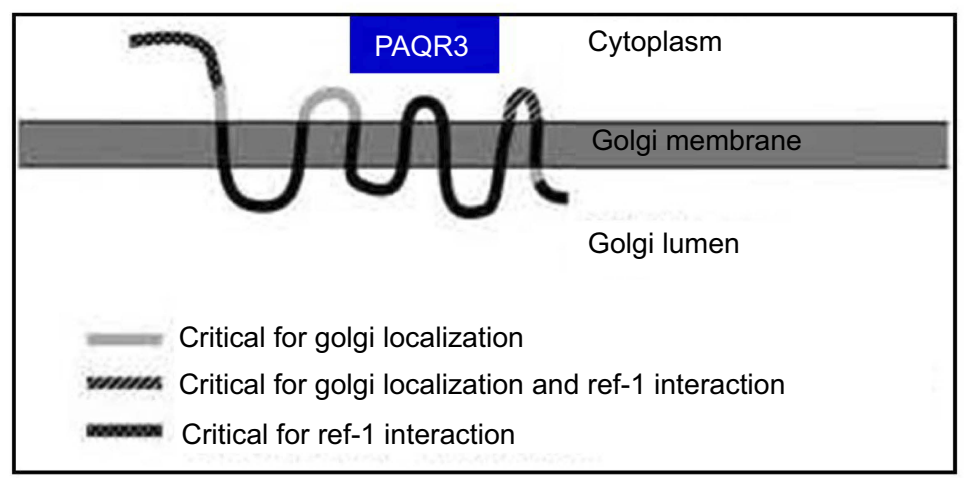

B

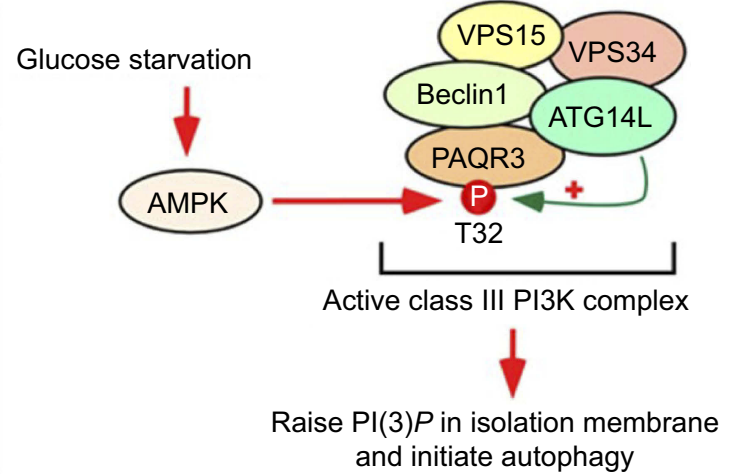

Figure I The topological model of PAQR3 and the autophagy pattern regulated by PAQR3 (A) PAQR3 is a seven-time transmembrane protein located on the Golgi body with N-terminal toward the cytoplasm amd C-terminal toward Golgi lumen. Feng L, Xie X, Ding Q, et al. Spatial regulation of Raf kinase signaling by RKTG. Proc Natl Acad Sci U S A. 2007;104(36): 14348-14353. Copyright (2007) National Academy of Sciences, U.S.A. ${ }^{32}$ (B) Activated AMPK phosphorylated PAQR3 at position 32 threonine during glucose starvation, promoting autophagy initiation, permission from EMBO. ${ }^{62}$ 
HLY3), the adiponectin receptor related PAQR gene members PAQR1 and PAQR2, the progesterone hormone membrane receptor PAQR gene members PAQR4 PAQR9. ${ }^{38}$

Like other PAQR genes, the PAQR3 gene of all human or mouse sources contains a highly conserved PFAMUPF0073 domain. According to the hydrophilic and hydrophobic analysis of the sequence, this domain is predicted to contain a seven-times transmembrane structure, but compared with the typical I transmembrane structure of GPCR (that is, the N-terminal toward the extracellular C-terminal to the intracellular plasma membrane protein), PFAM domain has a more complex and diverse topological structure. The structures of the identified membrane protein progesterone membrane receptors and adiponectin receptor members showed that their transmembrane region sequences were not similar to those of known GPCR genes. The results of cell composition analysis and immunofluorescence localization showed that PAQR3 is not located in plasma membrane like GPCR or known PAQR family membrane receptor members, but has the same distribution as Golgi membrane protein Golgi97,GM130 and is a Golgi cell membrane protein. Further study shows that PAQR3 is a type III topological seven-time transmembrane protein, with its $\mathrm{N}$ end facing cytoplasm side, $\mathrm{C}$ terminal is located in the Golgi cavity, and the N-end towards cytoplasmic side and Loop structure are mainly responsible for binding a series of signal molecules (including BRaf, C-Raf kinase) in cytoplasm to influence the signal transmission and output of intracellular signal molecules (Figure 1A). ${ }^{33}$

\section{PAQR3 is a tumor suppressor in multiple cancers \\ Breast cancer}

The expression of PAQR3 is markedly reduced in human breast adenocarcinoma specimens compared with the adjacent non-cancerous tissues. ${ }^{11,39}$ Also, PAQR3 levels are inversely correlated with progression and survival in breast cancer patients, in addition to cell proliferation, cell migration and HER2 amounts in human breast adenocarcinoma cells. Meanwhile, PAQR3 overexpression reduces malignancy potential in human breast adenocarcinoma. ${ }^{11,39}$ Conversely, PAQR3 silencing in MDA-MB-231 cells promotes cell proliferation and migration. Abnormal methylation of the PAQR3 gene is one of the reasons behind its downregulation in breast cancer. Promoter hypermethylation may significantly affect the silencing of the epigenetic expression of the PAQR3 gene in breast cancer. ${ }^{39}$

HER2 is a critical target in breast cancer treatment. Breast adenocarcinoma is also treated by adjuvant or neoadjuvant chemotherapeutics in combination with HER2-targeted molecules, eg, trastuzumab. ${ }^{40}$ Recent studies have shown PAQR3 amounts are tightly associated with HER2 levels. ${ }^{11}$ A possibility is that HER2 downregulates PAQR3, indicating that modulating HER2 function controls PAQR3 expression in HER2-positive breast adenocarcinoma. PAQR3 downregulation mediated by HER2 might be crucial for enhancing the tumorigenic effects of HER2. Future studies should unveil the molecular mechanism underlying HER2-PAQR3 interaction in the tumorigenesis and progression of breast cancer, which might further clarify the carcinogenetic mechanism and help develop targeted therapy.

\section{Prostate cancer}

Accumulating evidence reveals PAQR3 suppresses human prostate cancer cell growth. ${ }^{41,42}$ It has been found PAQR3 inhibits malignant potential in prostate cancer in cell culture and animal models. ${ }^{42}$ 3-(4,5-Dimethylthiazol-2-yl)-2,5diphenyltetrazolium bromide (MTT) and colony formation assay showed that PAQR3 overexpression could inhibit PC3 and DU145 cell proliferation; as expected, PAQR3 silencing enhanced PC3 and DU145 cell proliferation. In addition, high PAQR3 amounts resulted in reduced PC3 and DU145 cell migration, which was promoted by PAQR3 silencing. In animals with xenografted tumors, PAQR3 overexpression was shown to inhibit prostate cancer PC3 cells, with PAQR3 silencing having opposite effects. Furthermore, PAQR3 inhibited serum-associated Akt and ERK phosphorylation in PC3 and DU145 cells, while repressing EMT in PC3 cells. Mechanistically, it could inhibit both PI3K/Akt and Ras/Raf/MEK/ERK pathways in prostate cancer cells, which might account for its anticancer effects. It has been reported that promoter methylation of PAQR3 is more frequently encountered in prostate cancer in comparison with benign prostatic hyperplasia (BPH) (73.9\% vs $25.8 \%, P<0.01$ ), and suggested that PAQR3 methylation could be used as a promising marker for detecting and monitoring prostate cancer. ${ }^{41}$

\section{Esophageal squamous cell carcinoma cells} It has been demonstrated that PAQR3 amounts are markedly reduced in esophageal squamous cell carcinoma (ESCC) compared with the adjacent noncancerous tissue. 
In addition, the lower the PAQR3 expression, the more elevated the TNM stage and the greater the lymph node involvement. Moreover, PAQR3 was shown to independently predict prognosis in ESCC. PAQR3 amounts were remarkably reduced in ESCC cell lines compared with non-cancerous esophageal epithelial cells. 5-Aza-2 -deoxycytidine (5-Aza-CdR) administration resulted in PAQR3 upregulation in ESCC cells, inhibiting their malignant potential. In addition, cell cycle blockage occurred in the G1 phase, in association with p27 and p21 upregulation and cyclin D1, CDK4, and CDK2 downregulation. Moreover, it also suppressed ECA-109 xenograft tumors in animals. Mechanistically, overexpression of PAQR3 suppressed the ERK pathway. ${ }^{43,44}$ And, it was found that PAQR3 is lowly expressed in human ESCC cell lines. PAQR3 overexpression was shown to reduce malignant potential in cultured ESCC cells and attenuated the growth of xenografted tumors. Mechanistically, it suppressed the expression of RAF1, p-MEK1, and p-ERK1/2 in ESCC cells. ${ }^{45}$ Jointly, the above findings demonstrated PAQR3 overexpression suppresses the aggressive phenotype of ESCC cells in culture and animals, indicating PAQR3 could be targeted for ESCC treatment. ${ }^{43-45}$

\section{Gastric cancer}

PAQR3, a novel genetic signature, predicts prognosis in GC. It has been revealed that PAQR3 downregulation in human gastric cancer (GC), which was negatively correlated with Helicobacter pylori infection and remarkably correlated with GC malignancy. PAQR3 level reduction is closely associated with induced EMT in GC cells. Meanwhile, overexpression of PAQR3 could suppress cell growth, migration, and EMT in GC cells. ${ }^{35}$ Mechanistically, PAQR3 enhances Twist1 degradation to inhibit EMT and metastasis in GC cells. ${ }^{46}$ Other study reported that DDB2 (damage-specific DNA-binding protein 2) participates in PAQR3 ubiquitination and degradation, regulating GC cell tumorigenesis. ${ }^{47}$

In addition, a study demonstrated that miR-15b-5p is highly expressed in GC. Indeed, elevated plasma levels of miR-15b-5p might be used as a biomarker of GC metastasis. Mechanistically, miR-15b-5p overexpression enhances GC metastasis by controlling PAQR3 expression. ${ }^{48}$

\section{Colorectal cancer}

PAQR3 was found to be a tumor suppressor in colorectal cancer. ${ }^{49}$ In cells, PAQR3 controls proliferation, anchorage-independent growth, ERK phosphorylation, and nuclear accumulation of $\beta$-catenin in colorectal malignant SW-480 cells. In Apc ${ }^{\mathrm{Min} /+}$ mice, Paqr3 suppression enhances tumorigenesis. In humans, PAQR3 is markedly downregulated in colorectal cancer specimens in comparison with the corresponding non-cancerous tissue specimens. Meanwhile, PAQR3 downregulation in colon cancer is correlated with increased tumor grade. ${ }^{49}$ Another study confirmed the role of PAQR 3 gene methylation in colorectal cancer. The methylating rates of PAQR 3 in cancer tissues and paracancerous non-cancer tissue specimens were $33.3 \%(18 / 54)$ and $5.6 \%(3 / 54)$, respectively. ${ }^{50}$

\section{PAQR3 represents a new tumor suppressor gene}

PAQR3 is considered a tumor suppressor gene in breast cancer, prostate cancer, ESCC, GC, colorectal cancer, hepatocellular carcinoma, ${ }^{36,51}$ glioma,${ }^{52}$ leukemia,${ }^{53}$ etc. The details are summarized in Table 1.

\section{PAQR3 modulates metabolic homeostasis and participates in the compartmentalization of cell signaling}

PAQR3 has a critical function in metabolism. It suppresses insulin signaling via shunting of cytosolic p110 $\alpha$ to the Golgi and competition with the $\mathrm{p} 85$ subunit in generating the PI3K-p110 $\alpha$ complex. ${ }^{58}$ The expression of PAQR3 is changed in individuals with insulin resistance, and its suppression enhances insulin sensitivity. ${ }^{58}$ In addition, PAQR3 regulates obesity mediated by the leptin signaling pathway. ${ }^{59}$ In cholesterol metabolism, PAQR3 controls cholesterol homeostasis via anchoring of sterol regulatory element binding protein SREBP cleavage-activating protein (SCAP) to the Golgi, which reduces cholesterol biosynthesis. Consequently, disrupting PAQR3 interaction with SCAP/SREBP is considered a promising tool for reducing hepatic cholesterol synthesis. ${ }^{60}$ PAQR3 also is critical in regulating hepatic low density lipoprotein receptor (LDLR) degradation as well as circulating low density lipoprotein cholesterol (LDL-C) amounts by modulating LDLR-proprotein convertase subtilisin kexin type 9 (PCSK9) interaction, suggesting that blocking the fusion of PAQR3-containing vesicles with those carrying LDLR/ PCSK9 could help reduce blood cholesterol levels. ${ }^{61}$ Other studies have found that PAQR3 participates in autophagy initiation by two mechanisms: (1) enhancement of the ability of proautophagy class III PI3K using the scaffold 


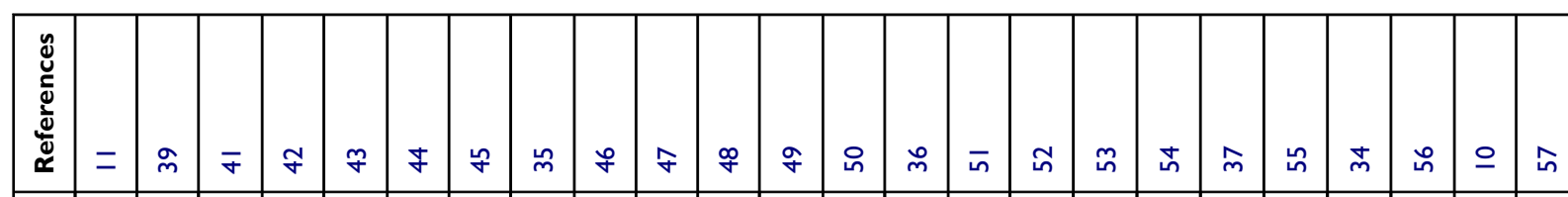

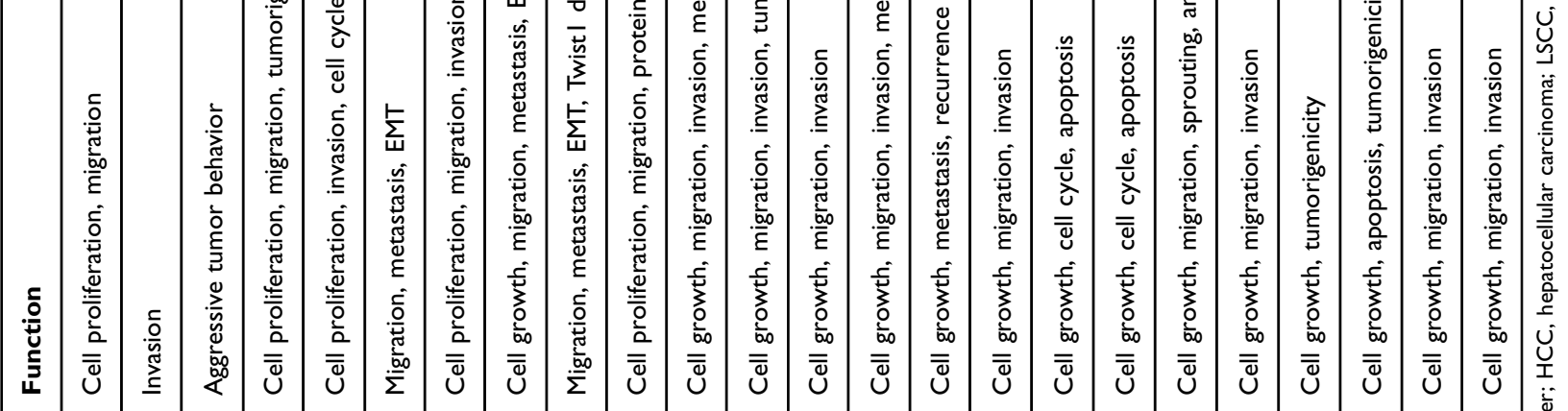

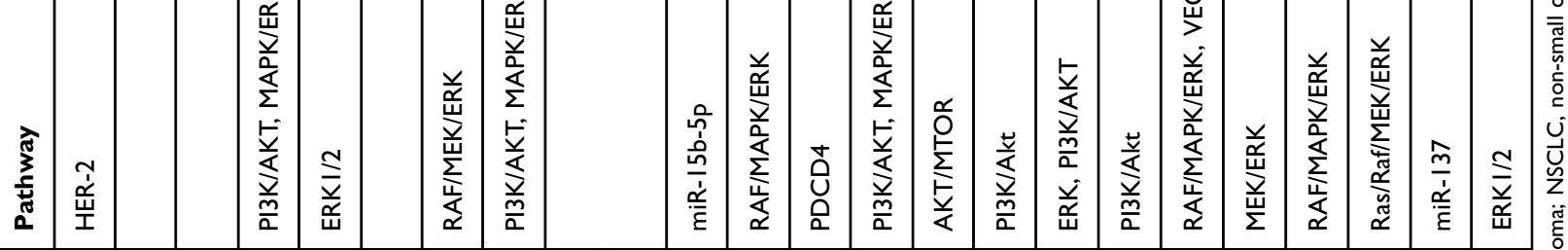

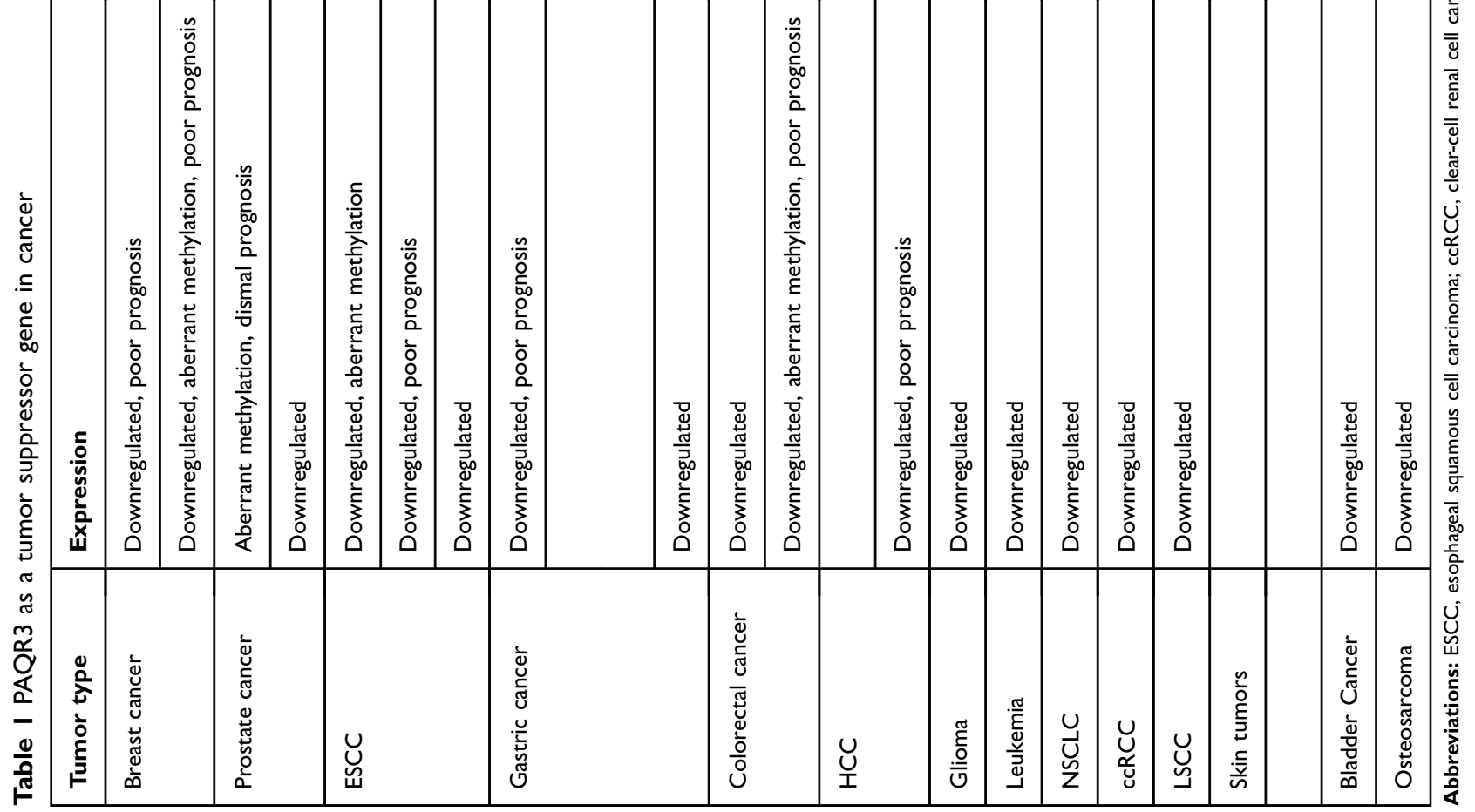


function; (2) AMPK signal integration into the activation of ATG14-bound VPS34 complex following glucose deprivation (Figure 1B). ${ }^{62,63}$ Another study provided crucial evidence that PAQR3 downregulates the mammalian target of rapamycin complex 1 (mTORC1) pathway, modulating autophagy in cultured cells and animal models in response to altered amino acid amounts. ${ }^{64}$

It has been found that the overexpression of PAQR3 upregulates fibronectin and intercellular adhesion molecule1 in glomerular mesangial cells grown in high glucose conditions, with PAQR3 controlling the formation of diabetes associated renal inflammatory fibrosis via nuclear factor kappa-B (NF- $\kappa \mathrm{B})$ signaling. ${ }^{65}$ Another study showed that the traditional Chinese medicine Tetramethylpyrazine, used for angina pectoris treatment, inhibits atherosclerosis progression and improves fat metabolism disorders via PAQR3 downregulation and SCAP/SREBP-1c signaling suppression in high-fat diet administered $\mathrm{ApoE}^{-/-}$mice, likely involving PI3K/Akt/mTORC1 signaling. ${ }^{66}$

\section{Molecular mechanisms of PAQR3 as a multifunctional regulator of diverse physiological processes}

The PAQR3 protein has a variety of functions, regulating a series of signaling pathways and physiological processes. In cultured tumor cells in vitro, PAQR3 interacts with Raf in Ras/ Raf/MEK/ERK signaling, anchoring it to the Golgi apparatus, thereby inhibiting this pathway and the development of tumors. ${ }^{32}$ PAQR3 is a new membrane protein located in the Golgi. PAQR3's N-terminus binds G $\beta$ and anchors $G \beta \gamma$ to the Golgi. PAQR3 upregulation suppresses G $\beta \gamma$ interaction with GRK2, thus eliminating the alteration in the distribution of ligand-induced GRK2 and reducing the phosphorylation of the isoproterenol receptor ( $\beta 2 \mathrm{AR}$ ) and its desensitization. Meanwhile, PAQR3 can also inhibit G $\beta \gamma$ - and ligand-associated Akt phosphorylation, which is induced by PAQR3 downregulation. PAQR3 silencing equally changes the internalization of GRK2 and ligand-induced G $\beta \gamma$ transport to the Golgi. In a word, PAQR3 modulates GPCR signaling via G $\beta \gamma$ targeting to the Golgi, so that its function can be reduced. ${ }^{9,67}$

Peroxisome proliferator-activated receptors (PPARs) are critical transcription factors regulating liver fat catabolism. They help adapt to nutritional starvation by inducing the oxidation of fatty acids as well as ketogenic production. PAQR3 is critical in regulating lipid catabolism in response to starvation via PPAR $\alpha$ modulation. ${ }^{68}$ PAQR3 interacts directly with PPAR $\alpha$ to increase para-polyubiquitination and proteasome- mediated degradation. Further, E3 ubiquitin ligase HECT, UBA and WWE domain containing E3 ubiquitin protein ligase 1 (HUWE1) mediates para-polyubiquitination. Moreover, PAQR3 enhances HUWE1-PPAR $\alpha$ interaction. ${ }^{68}$

Histone modifications are highly involved in several physiological processes, including development and tumorigenesis. Histone $\mathrm{H} 3$ lysine $4(\mathrm{H} 3 \mathrm{~K} 4)$ methylation is often involved in gene induction at the transcriptional level. H3K4 methylation in mammalians is performed by a protein complex related to Set1 (COMPASS)-like complexes comprising catalytic subunits, including mixed-lineage leukemia 1 (MLL1) and many modulatory subunits, with WD40 repeatcontaining protein 5 (WDR5), retinoblastoma-binding protein 5 (RBBP5), absent, small or homoeotic discs 2 (ASH2), and DPY30 [making up the WRAD sub-complex (WDR5-ASH2RBBP5-DPY30 complex)] as main entities found in both yeasts and metazoans. H3K4 methylation is controlled by PAQR3 spatially through alterations in subcellular compartmentalization of the WRAD subcomplex in mammalians. ${ }^{69}$

A study revealed PAQR3 equally plays an important role in repairing DNA damage by functionally altering the repair protein RAD23 homolog B (RAD23B)- Xeroderma pigmentosum group $\mathrm{C}$ (XPC). ${ }^{70}$ Another study pinpointed PAQR3 constitutes an adaptor molecule that promotes nuclear factor erythroid 2 [NF-E2]-related factor 2 (Nrf2)-Kelch-like erythroid cell-derived protein with $\mathrm{CNC}$ homology [ECH]-associated protein 1 (KEAP1) complex generation, thereby modulating the related pathway and controlling cellular antioxidant reactions. ${ }^{71}$

A recent study found that PAQR3's N-terminus interacts with the WD domains of Sec13 and Sec31A, enhancing their Golgi accumulation. This finding revealed that PAQR3 is a key molecule that regulates ER-to-Golgi translocation via interaction with the Sec13/Sec31A coat protein complex of COPII vesicles. ${ }^{72}$

\section{Different regulatory functions of PAQR3 in multiple signaling pathways}

PAQR3 is highly involved in the regulation of energy metabolism, cell homeostasis maintenance, and cell survival promotion. It can sense and integrate signaling factors such as nutrition and oxygen concentrations. Different response mechanisms are selected according to the tissue microenvironment, as the active adaptation of the organism to changes in the internal microenvironment during the long-term evolution process. Findings on various 
regulatory functions of PAQR3 in multiple signaling pathways are shown in Figure $2 .^{73}$

There is a research group synthetically produced a peptide simulating the suppressive effects of PAQR3, and assessed PAQR3's effects on human gastric cancer in cell and animal models. These authors demonstrated that blockade of the interaction between $\mathrm{p} 110 \alpha$ and P85 using the synthetic peptide based on PAQR3 constitutes a novel tool for inhibiting class I PI3K and decreasing the carcinogenic effects of PI3K. This finding provides an objective experimental basis for treating gastric cancer with PAQR3 targeting PI3K. ${ }^{74}$

\section{Summary and outlook}

In conclusion, PAQR3, as a Golgi membrane protein, can inhibit the activation of Raf/MEK/ERK signal pathway by binding B-Raf and C-Raf to the upstream and downstream signals, thus affecting the expansion and transformation of cells, and the tumorigenesis and development of the body. At present, the study of PAQR3 gene function is still in its preliminary stage, whether at the molecular level or in animal models to reveal its physiological function may only be the tip of the iceberg. In the mechanism of action, the signal pathway regulated by PAQR3 needs to be further investigated. For example, what is the mechanism that PAQR3 affects the HER2 when the effect of PAQR3 on the HER2 signal has been found in breast cancer? PAQR3 must have more binding protein on the Golgi, then what kind of way is it to control the signal pathway and physiological function of these binding proteins? What is the effect of the modification of phosphorylation

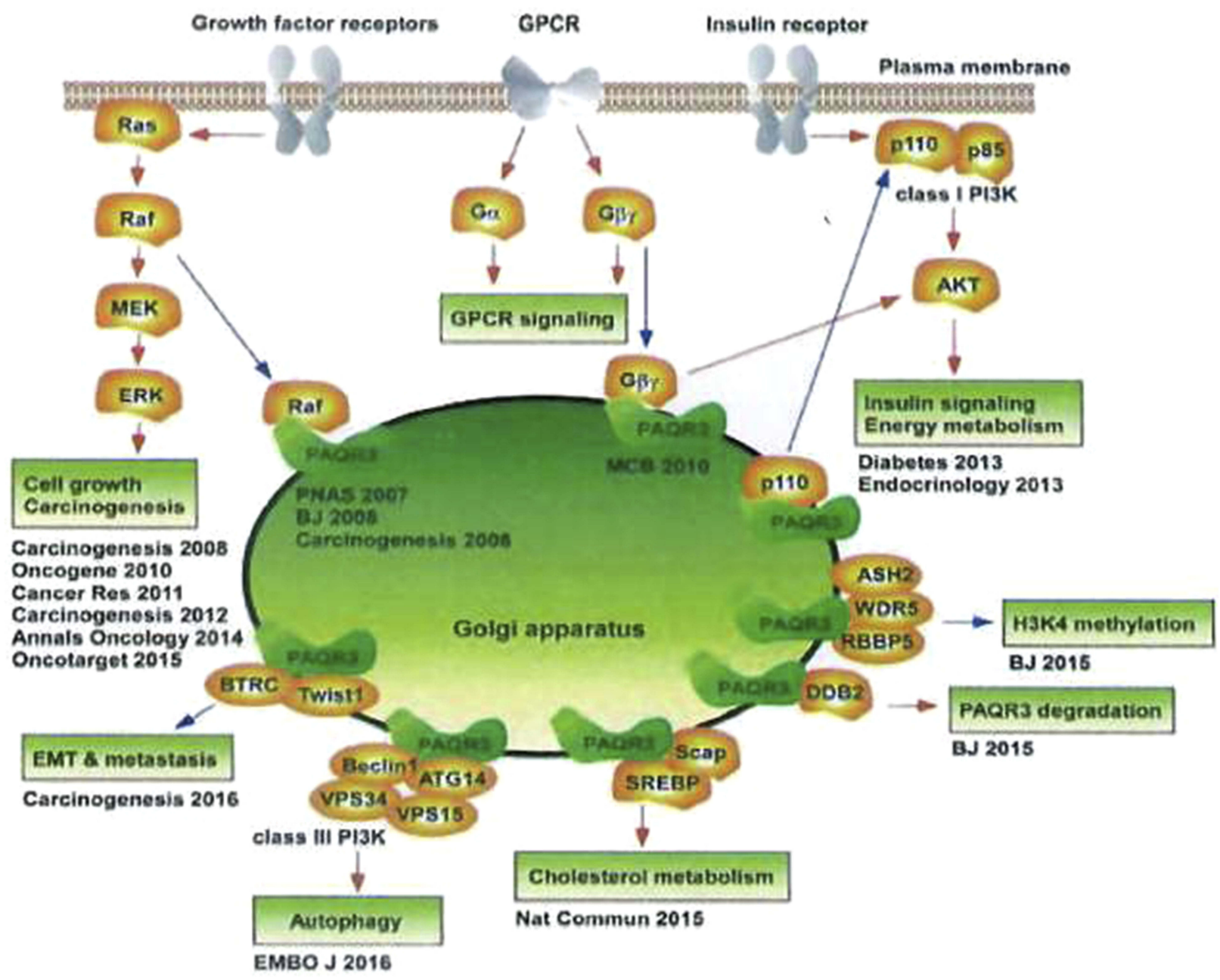

Positive regulation

\section{Negative regulation}

Figure 2 Different regulation of PAQR3 on multiple signaling pathways.

Notes: Reproduced from Zhao Z, Chen Y. Post-translational regulation of PAQR3 on PPAR $\alpha$, a critical transcription factor controlling fatty acid metabolism in the liver. A dissertation submitted to University of Chinese Academy of 695 Sciences (CAS) in partial fulfillment of the requirement for the degree of Doctor of Philosphy in Biochemistry and Molecular Biology by Zhao Ziling. Supervisor: Chen Yan. Shanghai Inst Biol Sci CAS. 2018;6:8-10. ${ }^{73}$ 
sites of Raf kinase on its binding to PAQR3? Does Raf kinase anchored on Golgi apparatus by PAQR3 have special physiological functions? What is the effect of PAQR3 on cancer caused by other cancers and other factors at the animal or body level? In a word, the true physiological role of PAQR3 in human tumors, metabolism, or other diseases is far from being fully understood.

In addition, the molecules of different subpopulations of the PAQR family play a regulatory role in the processes of fatty acid oxidation, inflammation, metabolism, cell apoptosis, tumorigenesis, sex hormone regulation, etc. The study of the PAQR3 family, including the PAQR3, is gradually increasing, but the indirect estimation of its biological function is more and more, and there are still large disputes in many aspects. Due to the diversity of PAQR family members in the subcellular localization and distribution characteristics, there is a lack of functional contact between the members of the family. With the indepth study of the members of the PAQR family, on the one hand, the understanding of its existing physiological and pathological processes will be updated, on the other hand, the signal transduction pathway mediated by this kind of protein will be more clear, which will provide new targets and strategies for the treatment of tumors and metabolic related diseases.

\section{Acknowledgments}

This work was funded by the National Natural Science Foundation of China (81372332 and 81572822), key projects from the Natural Science Foundation of Zhejiang Province of China (LZ13H160002 and LZ18H160002), the National Health and Family Planning Commission Scientific Research Foundation-Zhejiang Medical and Health Major Science and Technology Plan (WKJ-ZJ1505), the Leading Talents in Scientific and Technological Innovation from Zhejiang Provincial Ten Thousand Talents Plan (Zhejiang Provincial CPC Committee Talents [2019]3), Zhejiang Provincial Program for the Cultivation of High-Level Innovative Health Talents (Zjwjw2014-108), and the Major Training Personnel from Zhejiang Provincial Program for the Training and Development Project for 151 Talents (Zjhrss2014-150).

\section{Disclosure}

The authors report no conflicts of interest in this work.

\section{References}

1. Allemani C, Coleman MP. Public health surveillance of cancer survival in the United States and worldwide: the contribution of the CONCORD programme. Cancer. 2017;123(Suppl 24):4977-4981. doi:10.1002/cncr.v123.S24

2. Johnson CE, Tee AR. Exploiting cancer vulnerabilities: mTOR, autophagy, and homeostatic imbalance. Essays Biochem. 2017;61 (6):699-710. doi:10.1042/EBC20170056

3. Kim JA, Yeom YI. Metabolic signaling to epigenetic alterations in cancer. Biomol Ther (Seoul). 2018;26(1):69-80. doi:10.4062/ biomolther.2017.185

4. Kumar A, Placone JK, Engler AJ. Understanding the extracellular forces that determine cell fate and maintenance. Development. 2017;144(23):4261-4270. doi:10.1242/dev. 158469

5. Jain BP. An overview of unfolded protein response signaling and its role in cancer. Cancer Biother Radiopharm. 2017;32(8):275-281. doi:10.1089/cbr.2017.2309

6. Peng W, Lei Q, Jiang Z, et al. Characterization of Golgi scaffold proteins and their roles in compartmentalizing cell signaling. $J \mathrm{Mol}$ Histol. 2014;45(4):435-445. doi:10.1007/s10735-013-9560-1

7. Jin T, Ding Q, Huang H, et al. PAQR10 and PAQR11 mediate Ras signaling in the Golgi apparatus. Cell Res. 2012;22(4):661-676. doi:10.1038/cr.2011.161

8. Yu X, Li Z, Chan MT, Wu WKK. PAQR3: a novel tumor suppressor gene. Am J Cancer Res. 2015;5(9):2562-2568.

9. Hewavitharana T, Wedegaertner PB. PAQR3 regulates Golgi vesicle fission and transport via the G $\beta \gamma-\mathrm{PKD}$ signaling pathway. Cell Signal. 2015;27(12):2444-2451. doi:10.1016/j.cellsig.2015.08.017

10. Xiu Y, Liu Z, Xia S, et al. MicroRNA-137 upregulation increases bladder cancer cell proliferation and invasion by targeting PAQR3. PLoS One. 2014;9(10):e109734. doi:10.1371/journal.pone.0109734

11. Li Z, Ling ZQ, Guo W, et al. PAQR3 expression is downregulated in human breast cancers and correlated with HER2 expression. Oncotarget. 2015;6(14):12357-12368. doi:10.18632/oncotarget.3657

12. Tang YT, Hu T, Arterburn M, et al. PAQR proteins: a novel membrane receptor family defined by an ancient 7-transmembrane pass motif. J Mol Evol. 2005;61(3):372-380. doi:10.1007/s00239-0040375-2

13. Villa NY, Moussatche P, Chamberlin SG, Kumar A, Lyons TJ. Phylogenetic and preliminary phenotypic analysis of yeast PAQR receptors: potential antifungal targets. J Mol Evol. 2011;73(34):134-152. doi:10.1007/s00239-011-9462-3

14. Villa NY, Kupchak BR, Garitaonandia I, et al. Sphingolipids function as downstream effectors of a fungal PAQR. Mol Pharmacol. 2009;75 (4):866-875. doi:10.1124/mol.108.049809

15. Gonzalez-Velazquez W, Gonzalez-Mendez R, Rodriguez-del Valle N. Characterization and ligand identification of a membrane progesterone receptor in fungi: existence of a novel PAQR in Sporothrix schenckii. BMC Microbiol. 2012;12:194. doi:10.1186/1471-218012-194

16. Zhu D, Zhang J, Bin Y, Xu C, Shen J, Matsuo M. Dielectric studies on the heterogeneity and interfacial property of composites made of polyacene quinone radical polymers and sulfonated polyurethanes. $J$ Phys Chem A. 2012;116(9):2024-2031. doi:10.1021/jp212446n

17. Svensk E, Stahlman M, Andersson CH, et al. PAQR-2 regulates fatty acid desaturation during cold adaptation in C. elegans. PLoS Genet. 2013;9(9):e1003801. doi:10.1371/journal.pgen.1003801

18. Pilon M, Svensk E. PAQR-2 may be a regulator of membrane fluidity during cold adaptation. Worm. 2013;2(4):e27123. doi:10.4161/ worm. 27123

19. Petersen SL, Intlekofer KA, Moura-Conlon PJ, et al. Novel progesterone receptors: neural localization and possible functions. Front Neurosci. 2013;7:164. doi:10.3389/fnins.2013.00164 
20. Morrill GA, Kostellow AB, Gupta RK. A computational analysis of non-genomic plasma membrane progestin binding proteins: signaling through ion channel-linked cell surface receptors. Steroids. 2013;78 (12-13):1233-1244. doi:10.1016/j.steroids.2013.08.006

21. Petersen SL, Intlekofer KA, Moura-Conlon PJ, et al. Nonclassical progesterone signalling molecules in the nervous system. $J$ Neuroendocrinol. 2013;25(11):991-1001. doi:10.1111/jne.12060

22. Ndiaye K, Poole DH, Walusimbi S, et al. Progesterone effects on lymphocytes may be mediated by membrane progesterone receptors. J Reprod Immunol. 2012;95(1-2):15-26. doi:10.1016/j.jri.2012.04.004

23. Charles NJ, Thomas P, Lange CA. Expression of membrane progesterone receptors $(\mathrm{mPR} / \mathrm{PAQR})$ in ovarian cancer cells: implications for progesterone-induced signaling events. Horm Cancer. 2010;1 (4):167-176. doi:10.1007/s12672-010-0023-9

24. Góñez LJ, Naselli G, Banakh I, Niwa H, Harrison LC. Pancreatic expression and mitochondrial localization of the progestin-adipoQ receptor PAQR10. Mol Med. 2008;14(11-12):697-704. doi:10.2119/ 2008-00072.Gonez

25. Flock GB, Cao X, Maziarz M, Drucker DJ. Activation of enteroendocrine membrane progesterone receptors promotes incretin secretion and improves glucose tolerance in mice. Diabetes. 2013;62(1):283290. doi:10.2337/db12-0601

26. Wellbrock C, Karasarides M, Marais R. The RAF proteins take centre stage. Nat Rev Mol Cell Biol. 2004;5(11):875-885. doi:10.1038/nrm1498

27. Kolch W. Coordinating ERK/MAPK signalling through scaffolds and inhibitors. Nat Rev Mol Cell Biol. 2005;6(11):827-837. doi:10.1038/ nrm1743

28. Rapp UR, Gotz R, Albert S. BuCy RAFs drive cells into MEK addiction. Cancer Cell. 2006;9(1):9-12. doi:10.1016/j.ccr.2005.12.022

29. Chappell WH, Steelman LS, Long JM, et al. Ras/Raf/MEK/ERK and $\mathrm{PI} 3 \mathrm{~K} / \mathrm{PTEN} / \mathrm{Akt} / \mathrm{mTOR}$ inhibitors: rationale and importance to inhibiting these pathways in human health. Oncotarget. 2011;2(3):135164. doi:10.18632/oncotarget. 240

30. Appleton CT, Usmani SE, Mort JS, Beier F. Rho/ROCK and MEK/ ERK activation by transforming growth factor-alpha induces articular cartilage degradation. Lab Invest. 2010;90(1):20-30. doi:10.1038/ labinvest.2009.111

31. Li Z, Shen J, Wu WK, et al. Leptin induces cyclin D1 expression and proliferation of human nucleus pulposus cells via JAK/STAT, PI3K/ Akt and MEK/ERK pathways. PLoS One. 2012;7(12):e53176. doi:10.1371/journal.pone.0053176

32. Feng L, Xie X, Ding Q, et al. Spatial regulation of Raf kinase signaling by RKTG. Proc Natl Acad Sci U S A. 2007;104 (36):14348-14353. doi:10.1073/pnas.0701298104

33. Luo X, Feng L, Jiang X, et al. Characterization of the topology and functional domains of RKTG. Biochem J. 2008;414(3):399-406. doi:10.1042/BJ20080948

34. Fan F, Feng L, He J, et al. RKTG sequesters B-Raf to the Golgi apparatus and inhibits the proliferation and tumorigenicity of human malignant melanoma cells. Carcinogenesis. 2008;29(6):1157-1163. doi:10.1093/carcin/bgn119

35. Ling ZQ, Guo W, Lu XX, et al. A Golgi-specific protein PAQR3 is closely associated with the progression, metastasis and prognosis of human gastric cancers. Ann Oncol. 2014;25(7):1363-1372. doi:10.1093/ annonc/mdu168

36. Jiang YH, Xie XD, Li ZG, et al. Functional cooperation of RKTG with p53 in tumorigenesis and epithelial-mesenchymal transition. Cancer Res. 2011;71(8):2959-2968. doi:10.1158/0008-5472.CAN-10-4077

37. Zhang Y, Jiang X, Qin X, et al. RKTG inhibits angiogenesis by suppressing MAPK-mediated autocrine VEGF signaling and is downregulated in clear-cell renal cell carcinoma. Oncogene. 2010;29(39):5404-5415. doi:10.1038/onc.2010.270

38. Thomas P, Pang Y, Dong J, et al. Steroid and G protein binding characteristics of the seatrout and human progestin membrane receptor alpha subtypes and their evolutionary origins. Endocrinology. 2007;148(2):705-718. doi:10.1210/en.2006-0974
39. Chen J, Wang F, Xu J, et al. The role of PAQR3 gene promoter hypermethylation in breast cancer and prognosis. Oncol Rep. 2016;36 (3):1612-1618. doi:10.3892/or.2016.4951

40. Romond EH, Perez EA, Bryant J, et al. Trastuzumab plus adjuvant chemotherapy for operable HER2-positive breast cancer. $N$ Engl J Med. 2005;353(16):1673-1684. doi:10.1056/NEJMoa052122

41. Lounglaithong K, Bychkov A, Sampatanukul P. Aberrant promoter methylation of the PAQR3 gene is associated with prostate cancer. Pathol Res Pract. 2018;214(1):126-129. doi:10.1016/j.prp.201 7.10.010

42. Huang W, Guo W, You X, et al. PAQR3 suppresses the proliferation, migration and tumorigenicity of human prostate cancer cells. Oncotarget. 2016;8(33):53948-53958. doi:10.18632/oncota rget.9807

43. Bai G, Yang M, Zheng C, et al. PAQR3 overexpression suppresses the aggressive phenotype of esophageal squamous cell carcinoma cells via inhibition of ERK signaling. Biomed Pharmacother. 2017;94:813-819. doi:10.1016/j.biopha.2017.07.154

44. Bai G, Yang M, Zheng C, Zhang L, Eli M. Suppressor PAQR3 associated with the clinical significance and prognosis in esophageal squamous cell carcinoma. Oncol Lett. 2018;15(4):5703-5711. doi: $10.3892 / \mathrm{ol} .2018 .8004$

45. Zhou F, Wang S, Wang J. PAQR3 inhibits the proliferation and tumorigenesis in esophageal cancer cells. Oncol Res. 2017;25:663671. doi:10.3727/096504016X14761384026719

46. Guo W, You X, Xu D, et al. PAQR3 enhances Twist1 degradation to suppress epithelial-mesenchymal transition and metastasis of gastric cancer cells. Carcinogenesis. 2016;37(4):397-407. doi:10.1093/carcin/ bgw013

47. Qiao S, Guo W, Liao L, et al. DDB2 is involved in ubiquitination and degradation of PAQR3 and regulates tumorigenesis of gastric cancer cells. Biochem J. 2015;469(3):469-480. doi:10.1042/BJ20150253

48. Zhao C, Li Y, Chen G, et al. Overexpression of miR-15b-5p promotes gastric cancer metastasis by regulating PAQR3. Oncol Rep. 2017;38 (1):352-358. doi:10.3892/or.2017.5673

49. Wang X, Li X, Fan F, et al. PAQR3 plays a suppressive role in the tumorigenesis of colorectal cancers. Carcinogenesis. 2012;33 (11):2228-2235. doi:10.1093/carcin/bgs245

50. Li RH, Zhang AM, Li S, et al. PAQR3 gene expression and its methylation level in colorectal cancer tissues. Oncol Lett. 2016;12 (3):1773-1778. doi:10.3892/ol.2016.4843

51. Wu HG, Zhang WJ, Ding Q, et al. Identification of PAQR3 as a new candidate tumor suppressor in hepatocellular carcinoma. Oncol Rep. 2014;32(6):2687-2695. doi:10.3892/or.2014.3532

52. Tang SL, Gao YL, Hu WZ. PAQR3 inhibits the proliferation, migration and invasion in human glioma cells. Biomed Pharmacother. 2017;92:24-32. doi:10.1016/j.biopha.2017.05.046

53. Xu Y, Deng N, Wang X, Chen Y, Li G, Fan H. RKTG overexpression inhibits proliferation and induces apoptosis of human leukemia cells via suppression of the ERK and PI3K/AKT signaling pathways. Oncol Lett. 2017;14(1):965-970. doi:10.3892/ol.2017.6182

54. Li X, Li M, Chen D, Shi G, Zhao H. PAQR3 inhibits proliferation via suppressing PI3K/AKT signaling pathway in non-small cell lung cancer. Arch Med Sci. 2018;14(6):1289-1297. doi:10.5114/aoms.2017.72220

55. Wu Q, Zhuang K, Li H. PAQR3 plays a suppressive role in laryngeal squamous cell carcinoma. Tumour Biol. 2016;37(1):561-565. doi:10.1007/s13277-015-3770-y

56. Xie X, Zhang Y, Jiang Y, et al. Suppressive function of RKTG on chemical carcinogen-induced skin carcinogenesis in mouse. Carcinogenesis. 2008;29(8):1632-1638. doi:10.1093/carcin/bgn139

57. Ma Z, Wang Y, Piao T, et al. The tumor suppressor role of PAQR3 in osteosarcoma. Tumour Biol. 2015;36(5):3319-3324. doi:10.1007/s13277014-2964-z

58. Wang X, Wang L, Zhu L, et al. PAQR3 modulates insulin signaling by shunting phosphoinositide 3-kinase p110 $\alpha$ to the Golgi apparatus. Diabetes. 2013;62(2):444-456. doi:10.2337/db12-0244 
59. Wang L, Wang X, Li Z, et al. PAQR3 has modulatory roles in obesity, energy metabolism, and leptin signaling. Endocrinology. 2013;154 (12):4525-4535. doi:10.1210/en.2013-1633

60. Xu D, Wang Z, Zhang Y, et al. PAQR3 modulates cholesterol homeostasis by anchoring Scap/SREBP complex to the Golgi apparatus. Nat Commun. 2015;6:8100. doi:10.1038/ncomms9100

61. Huang M, Zhao Z, Cao Q, et al. PAQR3 modulates blood cholesterol level by facilitating interaction between LDLR and PCSK9. Metabolism. 2019;94:pii: S0026-0495(19)30047-2.

62. Xu DQ, Wang Z, Wang CY, et al. PAQR3 controls autophagy by integrating AMPK signaling to enhance ATG14L-associated PI3K activity. EMBO J. 2016;35(5):496-514. doi:10.15252/embj. 201592864

63. Xu D, Wang Z, Chen Y. Two-layer regulation of PAQR3 on ATG14-linked class III PtdIns3K activation upon glucose starvation. Autophagy. 2016;12(6):1047-1048. doi:10.1080/15548627. 2016.1163459

64. Wang L, Pan Y, Huang M, et al. PAQR3 augments amino acid deprivation-induced autophagy by inhibiting mTORC1 signaling. Cell Signal. 2017;33:98-106. doi:10.1016/j.cellsig.2017.02.017

65. Zou Y, Chen Z, Li J, et al. Progestin and AdipoQ receptor 3 upregulates fibronectin and intercellular adhesion molecule-1 in glomerular mesangial cells via activating NF- $\mathrm{B}$ signaling pathway under high glucose conditions. Front Endocrinol (Lausanne). 2018;9:275. doi:10.3389/fendo.2018.00420

66. Zhang Y, Ren P, Kang Q, et al. Effect of tetramethylpyrazine on atherosclerosis and SCAP/SREBP-1c signaling pathway in $\mathrm{ApoE}^{-/-}$ Mice Fed with a high-fat diet. Evid Based Complement Alternat Med. 2017;2017:3121989. doi:10.1155/2017/3121989

67. Jiang Y, Xie X, Zhang Y, et al. Regulation of G-protein signaling by RKTG via sequestration of the $G$ betagamma subunit to the Golgi apparatus. Mol Cell Biol. 2010;30(1):78-90. doi:10.1128/MCB.01038-09
68. Zhao Z, Xu D, Wang Z, et al. Hepatic PPAR $\alpha$ function is controlled by polyubiquitination and proteasome-mediated degradation through the coordinated actions of PAQR3 and HUWE1. Hepatology. 2018;68(1):289-303. doi:10.1002/hep.29786

69. Liu C, Zhang Y, Hou Y, et al. PAQR3 modulates H3K4 trimethylation by spatial modulation of the regulatory subunits of COMPASSlike complexes in mammalian cells. Biochem J. 2015;467(3):415424. doi:10.1042/BJ20141392

70. You X, Guo W, Wang L, et al. Subcellular distribution of RAD23B controls XPC degradation and DNA damage repair in response to chemotherapy drugs. Cell Signal. 2017;36:108-116. doi:10.1016/j. cellsig.2017.04.023

71. Zhang Y, Hou Y, Liu C, et al. Identification of an adaptor protein that facilitates Nrf2-Keap1 complex formation and modulates antioxidant response. Free Radic Biol Med. 2016;97:38-49. doi:10.1016/j. freeradbiomed.2016.05.017

72. Cao Q, Wang Z, Wan $\mathrm{H}$, et al. PAQR3 regulates endoplasmic Reticulum-to- Golgi trafficking of COPII vesicle via interaction with Sec13/Sec31 coat proteins. iScience. 2018;9:382-398. doi:10.1016/j.isci.2018.11.002

73. Zhao Z, Chen Y. Post-translational regulation of PAQR3 on PPAR $\alpha$, a critical transcription factor controlling fatty acid metabolism in the liver. A dissertation submitted to University of Chinese Academy of Sciences (CAS) in partial fulfillment of the requirement for the degree of Doctor of Philosphy in Biochemistry and Molecular Biology by Zhao Ziling. Supervisor: Chen Yan. Shanghai Inst Biol Sci CAS. 2018;6:8-10.

74. Guo W, You X, Wang X, Wang L, Chen Y. A synthetic peptide hijacks the catalytic subunit of class I PI3K to suppress the growth of cancer cells. Cancer Lett. 2017;405:1-9. doi:10.1016/j.canlet.2017.07.015

\section{Publish your work in this journal}

Cancer Management and Research is an international, peer-reviewed open access journal focusing on cancer research and the optimal use of preventative and integrated treatment interventions to achieve improved outcomes, enhanced survival and quality of life for the cancer patient.
The manuscript management system is completely online and includes a very quick and fair peer-review system, which is all easy to use. Visit http://www.dovepress.com/testimonials.php to read real quotes from published authors. 